\title{
Modeling techniques: theory and practice $\dagger$
}

\author{
O. A. ASBJØRNSEN $\ddagger$
}

Keywords: Systems engineering, model structure, model variables, chemical processes, orthogonal decomposition, conservation principle, invariance, reactors.

A survey is given of some crucial concepts in chemical process modeling. Those are the concepts of physical unit invariance, of reaction invariance and stoichiometry, the chromatographic effect in heterogeneous systems, the conservation and balance principles and the fundamental structures of cause and effect relationships. As an example, it is shown how the concept of reaction invariance may simplify the homogeneous reactor modeling to a large extent by an orthogonal decomposition of the process variables. This allows residence time distribution function parameters to be estimated with the reaction in situ, but without any correlation between the estimated residence time distribution parameters and the estimated reaction kinetic parameters. A general word of warning is given to the choice of wrong mathematical structure of models.

\section{Introduction}

Mathematical modeling in chemical engineering has a very long tradition, practically speaking from the establishment of the profession. As in many other fields, there are two different approaches to modeling, one is based on a simple empirical (black box) approach to the analysis of observations (empirical regression analysis), and the other is based on a systematic and careful definition of the physical characterization of the process and a subsequent application of general basic principles.

In view of the fact that chemical engineering processes involve a very large number of interacting mechanisms, it is understandable that process technology applies a high degree of empiricism in its models and modeling techniques. However, this condition is gradually changing because modern computing techniques make it possible to add more sophisticated models to the empirical interpretation of data.

Furthermore, the empirical approach is more dangerous and far less general than the basic principle approach, because the structure of the empirical model may not comply with the structure and constraints of a model based on physical principles. A wrong model may very well be fitted to experimental data resulting in catastrophic extrapolations. A good example here is the modeling of binary vapourliquid equilibrium data. A purely empirical approach would probably assume an $n$th order polynomial (Ohno and Nakanishi 1985) which will not satisfy the absolute constraints (Asbjørnsen and Hertzberg 1974):

$$
y=\sum_{i=0}^{n} a_{i} x^{i}
$$

Received 15 August, 1985.

$\uparrow$ This paper was presented at the International Seminar on Modern Methods in Dynamic Simulation of Industrial Processes, Trondheim, Norway, May 1985

‡ University of Houston, Chem. Eng. Dept. 4800 Calhoun, Houston, Texas 77004, USA. 
While the theoretical and ideal relationship with constant relative volatility is a hyperbola which automatically satisfies the constraints $(0,0)$ and $(1,1)$ :

$$
y=\alpha(x) x /(1+(\alpha(x)-1) x) .
$$

If a polynomial should be used, it might be used to express the relative volatility and as a function of the liquid mole fraction $x$, while the main structure of the relationship is kept as a hyperbola.

It is amazing how ignorant many papers are of such simple conditions in mathematical modeling and how often basic phenomena in modeling are neglected. One such phenomenon is the highly oscillating coefficient values one gets when a polynomial is attempted to fit a non-polynomial function. This is an indication that the polynomial is the wrong type of functional model. In the example shown, the polynomial will show coefficients with very high magnitudes but with oscillating signs, because a hyperbola is being modeled with an integer exponent polynomial, which may be a divergent series.

Similar observations may be made in the analysis and modeling of chemical reactors. The stoichiometry of reactions always give linear combinations between some of the process variables, which are insensitive to the reactions. In fact, if those variables happened to be chosen as the observations for empirical models, one can see very clearly that the modeling of the reaction kinetic phenomena will not be possible through those variables, because they are reaction invariant.

Physical units, variable dimensions and dimensionless groups of variables are other conditions for mathematical modeling where several applications ignore the fact that most of the mathematical models used in chemical engineering are unit invariant. If a system is dimensionally correctly modeled, the system behaviour is uniquely defined by a set of combined physical variables which are unit invariant. Those combined variables may appear only in such combinations, and are usually referred to as dimensionless groups. In such a combination where only the combined variable may be observed, it is impossible to observe or separate one variable from the others simply by observation of the combined group. Therefore, the combined variables, or the dimensionless groups, are the only variables that should form the basis for mathematical modeling and variable correlations. If this is not done properly, one dimensionless variable may be correlated against another, where the correlation is simply a result of the unit invariance of the problem. The correlation does not reveal any phenomenological information about the process at all.

Many of the basic principles used in the mathematical modeling of chemical processes are universal, non-specific to chemical engineering. One example is the conservation principle which applies equally well to, for example, economics as to chemical engineering. Another example is the feedback principle and its stability analysis, which applies equally well to the solution of systems of semi-implicit equations as to recycle processes, as to process control, as to control engineering and cybernetics in general, as to social structures and human behaviour, etc. Again, it is astonishing to see how many papers and works in the various engineering disciplines consider the conservation principle and the feedback principle as unique to a special application, while indeed they are not.

It is therefore clear, that chemical engineering and process technology should be approached, taught and practised with a much stronger emphasis on phenomenological systems' engineering than is the case today. In current courses in chemical engineering, general principles are presented and used in such a large variety of 
disguises and transcriptions that they almost appear as new individual general principles. This is unfortunate, because a lot of rationalization and simplification of concepts could save time, effort and resources if used in the correct way.

In view of all the similarities and the general applicability of concepts thought of as being unique to chemical engineering, this paper tries to take the systems' engineering approach to process technology and chemical engineering. In this way, an attempt is made to focus the readers' attention to some forgotten, but nevertheless important generalities which may save time and mental effort for many practising engineers, for the teaching profession and the art of application.

\subsection{Analysis of process variables}

The first step in modeling is always associated with proper examination and understanding of the physical and chemical variables involved. This requires good insight, experience and education.

The process variables appear in different classes and definitions. They are basically thermodynamic state variables, physical properties and design variables for unit operations and apparati. A small fragment of these variables are measured directly, others may be inferred from these measurements. Modern on-line computation and statistical analysis have given the whole area of intelligent instrumentation and estimation of variables and parameters a strong push forwards. But, for a successful estimation, it is necessary to have a good and structurally correct model which can relate the variables and parameters in a proper way. Polynomials and linear relationships are not enough.

Process variables may be classified according to their appearance in the physical and mathematical model, in a process engineering context; a systems engineering context or a mathematical context. Table 1 shows a typical classification of this nature (Asbjørnsen, 1974).

Of special interest here is the classification of variables in the process engineering context as extensive and intensive variables. This has no direct parallel in systems' engineering as the extensive and intensive variables are thought of as having an apparently clear connection to thermodynamics. However, this is not exactly true, because specific quantities in general physics play the role of intensive variables in a more general sense. Intensive variables are point variables in mathematics and thermodynamics, while extensive variables are proportional to the extension of a thermodynamic system under consideration. Thermodynamics is indeed the crucial and fundamental science for process engineering, and forms the basis for all the mathematical modeling applied to this field of engineering.

\begin{tabular}{lcl}
\hline Process engineering & Systems' engineering & \multicolumn{1}{c}{ Mathematics } \\
\hline Disturbance & Input & Free, independent \\
Manipulator & Input & Free, independent \\
Response & Output & Dependent \\
Parameter & Parameter & Parameter \\
State & State & Dependent \\
Extensive & & Volume related \\
Intensive & & Point variable \\
\hline
\end{tabular}

Table 1. Classification of variables. 


\begin{tabular}{|c|c|c|}
\hline Process engineering & Systems' engineering & Mathematics \\
\hline Single variable & $\begin{array}{l}\text { Single input } \\
\text { Single output }\end{array}$ & Scalar \\
\hline $\begin{array}{l}\text { Multi-component } \\
\text { Multi-variable }\end{array}$ & $\begin{array}{l}\text { Multiple input } \\
\text { Multiple output } \\
\text { Single array }\end{array}$ & Vector \\
\hline Table & Multiple array & Matrix \\
\hline
\end{tabular}

Table 2. Types of process variables.

At first sight, it may seem that the concepts of extensive and intensive variables are specific to process engineering, but indeed they are not. They are general concepts in physics and mathematics. Even in economics the production rate or the volume of sales are extensive variables and unit price is an intensive or specific variable.

Furthermore, if one considers the concepts of state variables, they have a much wider definition and application than in thermodynamics. The equations of state in thermodynamics are traditionally confined to thermodynamic variables, and do not usually include states like velocity, acceleration, momentum, direction, etc. The process variables also appear in different types as for example, logical or boolean, natural or integer, floating point or real and even alphanumerical, string or text. They may also be classified according to the structure of groups of variables as in Table 2.

In process engineering, a multi-component mixture is characterized by a complete and unique set of thermodynamic states, like composition and energy, and these states are grouped into a vector. The sensitivities of the process responses to changes in the input, may be grouped into a table or a matrix, as shown later. Simple, single function relations between one variable and a lot of others may be described as a relation:

$$
x=f(\mathbf{u}, \mathbf{v})
$$

A vector of functions is then logically

$$
\mathbf{x}=\mathbf{f}(\mathbf{u}, \mathbf{v})
$$

And the sensitivity in $\mathbf{x}$ to changes in $\mathbf{u}$ and $\mathbf{v}$ is equally logically, described by the Jacobian matrix:

$$
J_{u}=\partial \mathbf{f} / \partial \mathbf{u} \text { and } J_{v}=\partial \mathbf{f} / \partial \mathbf{v}
$$

Even if a simple analysis of variables seems trivial, it is nevertheless of great importance to keep the simple classification of the appearance and type of the variables in mind. The form of the appearance of variables is important information in order to evaluate in which equation of the model structure the variables may appear, and then in what form (input, output or parameter). This again is useful information for the choice of the solution algorithm of the model equations. The type of the variable (scalar, vector or matrix) is important information for the structuring of the database system into which the numerical values of all the variables are supposed to enter during a calculation and solution of the model equations, or a general simulation. 


\section{Dimensional analysis and unit invariance}

All process variables are given physical units and dimensions, based on a set of elementary physical units. These may be basic units, for example for mass, length and time. If there are $n$ physical variables and $m$ basic units, it is clear that $n-m$ dimensionless, unit invariant groups or transformed variables may be formed. The relationship between all the basic units, the physical variables and the unit invariant groups, may be analysed very simply by taking the formal logarithm of all variable units. Define:

$\mathbf{u}=$ vector of logarithms of the basic physical units.

$\mathbf{x}=$ vector of logarithms of the physical variable units.

$A=$ matrix of exponents of the basic units in the physical variable units.

$N=$ matrix of exponents of the physical variables in the dimensionless variables.

The physical variable units are written in terms of the basic units:

$$
\mathbf{x}=A \mathbf{u} ; \quad \operatorname{dim}(A)=n \times m
$$

and the dimensionless groups in terms of the physical variables:

$$
N \mathbf{x}=N A \mathbf{u}=\mathbf{0} ; \quad \operatorname{dim}(N)=(n-m) \times n
$$

If the basic units are properly selected, the matrix $A$ has full rank $m$. Then, this matrix may be partitioned into an invertible part, $A_{1}$, and the $A_{2}$. Consequently, the matrix $N$ is partitioned accordingly:

$$
N A=N_{1} A_{1}+N_{2} A_{2}
$$

Since equation (7) must be satisfied for all $\mathrm{u}$, this means that the matrix $N$ and $A$ must be orthogonal, which again leads to the relationship:

$$
N A=0 \quad \text { or } \quad N_{1}=-N_{2} A_{2} A_{1}^{-1}
$$

This shows that the matrix $N_{2}$ may be chosen arbitrarily, which is equivalent to selecting a set of $n-m$ of the basic dimensionless groups. Let $N_{2}=I$, then:

$$
N_{1}=A_{2} A_{1}^{-1}
$$

Take as an example, the turbulent pressure drop in pipes, where it may be assumed that six variables are important, $p=$ pressure drop, $v=$ velocity, $d=$ diameter, $l=$ length,$\rho=$ density,$g=$ local acceleration of gravity. The basic units are $M=$ mass, $T=$ time and $L=$ length. Then the matrices are:

$$
A_{1}=\left[\begin{array}{rrr}
1 & -2 & 1 \\
0 & -1 & 1 \\
0 & 0 & 1
\end{array}\right] ; \quad A_{2}=\left[\begin{array}{rrr}
0 & 0 & 1 \\
1 & 0 & -3 \\
0 & -2 & 1
\end{array}\right]
$$

and:

$$
N=\left[\begin{array}{rrrrrr}
0 & 0 & -1 & 1 & 0 & 0 \\
-1 & 2 & 0 & 0 & 1 & 0 \\
0 & -2 & 1 & 0 & 0 & 1
\end{array}\right]
$$

which gives the dimensionless variables:

$$
z_{1}=l / d \quad z_{2}=v^{2} \rho / p, \quad z_{3}=d g / v^{2}
$$

well known as a geometrical ratio, a momentum ratio and a velocity ratio. 
Assume now that the viscosity is included as a physical variable of significance. Then:

$A_{2}=\left[\begin{array}{rrr}0 & 0 & 1 \\ 1 & 0 & -3 \\ 0 & -2 & 1 \\ 1 & -1 & -1\end{array}\right]$ and $N=\left[\begin{array}{rrrrrrr}0 & 0 & -1 & 1 & 0 & 0 & 0 \\ -1 & 2 & 0 & 0 & 1 & 0 & 0 \\ 0 & -2 & 1 & 0 & 0 & 1 & 0 \\ -1 & 1 & -1 & 0 & 0 & 0 & 1\end{array}\right]$

which gives the additional dimensionless variable:

$$
z_{4}=\mu v /(\rho \cdot d)
$$

a viscous shear stress to pressure ratio. By comparing the dimensionless variables, it is clearly seen that the ratio: $z_{2} / z_{4}=R e$ is the traditional Reynolds number.

This analysis and the example show in general, that a system may be modeled by $n-m$ variables $z$ of dimensionless units where $n$ is the number of physical variables, and $m$ the number of basic units. The relationship between the dimensionless variables $\mathbf{z}$ and the physical variables $\mathbf{y}$ is such that:

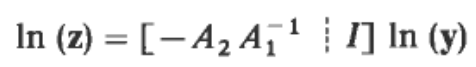

where $A$ is the matrix of exponents of basic units defining the units of the physical variables. The unit invariance is an absolute equality constraint on the variables.

\section{A qualitative modeling of cause and effect}

The traditional industrial analysis of process control systems on the design stage was based on a fairly simple analysis and classification of process variables, followed by a qualitative examination of the cause and effect relationship. This analysis and examination used qualitative statements from expert insights into process behaviour. This is in fact analogous to the modern concepts of expert systems, and was the first application of expert systems in process control system design about 25 years ago. Some of the early and systematic uses of this concept are found in a UNESCO report from the University of the West Indies at St. Augustine (Asbjørnsen, 1966) and in a textbook by Shinskey (Shinskey, 1967).

The expert statements are classified as if then statements of a qualitative nature, followed by qualitative statements on the speed of response of the process. The information gathered from such statements were concentrated and systematized in a table or a matrix, (Himmelblau 1978) the process matrix, which could be used as a guideline for single loop control, cascade and ratio control etc. The qualitative statements were as follows:

(1) If variable $u$ increases then variable $x$ will increase $(+)$

(2) If variable $u$ increases then variable $x$ will decrease (-)

(3) If variable $u$ changes then variable $x$ is unaffected(0)

(4) If variable $u$ changes then the effect on $x$ is unknown(?)

(5) The speed of response is known to be slow, normal or fast

With these statements, the process reactions are mapped in a table, or matrix, expressing the input output relationships qualitatively: 


\begin{tabular}{c|c|c|c|c}
\hline & $x_{1}$ & $x_{2}$ & $x_{3}$ & $x_{4}$ \\
\hline$u_{1}$ & $\begin{array}{c}+ \\
\text { fast }\end{array}$ & 0 & $\begin{array}{c}- \\
\text { slow }\end{array}$ & $?$ \\
\hline$u_{2}$ & $\begin{array}{c}+ \\
\text { normal }\end{array}$ & $\begin{array}{c}- \\
\text { slow }\end{array}$ & $\begin{array}{c}+ \\
\text { normal }\end{array}$ & 0 \\
\hline$u_{3}$ & 0 & $?$ & $\begin{array}{c}+ \\
\text { fast }\end{array}$ & $?$ \\
\hline$u_{4}$ & $\begin{array}{c}+ \\
\text { slow }\end{array}$ & normal & 0 & 0 \\
\hline
\end{tabular}

This matrix was used to pair variables in single feedback loops, according to a few rather simple rules

(1) Each controller introduced a new input, the set-point. This was then subject to similar expert statements, and regarded as a manipulator. It could be used further in cascaded loops.

(2) When pairing variables for control the sign of the controller should be the opposite of the corresponding sign in the process matrix for negative feedback control.

(3) Always pair fast variables as inner loops.

(4) Avoid pairing loops with question marks. If this is desirable, get additional expert advice.

Clearly, this qualitative approach to process modeling is not sufficient today, more quantitative information and process description will be required. However, as a first hand tool, this simple approach has solved many practical industrial control problems, and the solution has been very effective (Norsk Hydro, 1980).

\section{The structure of mathematical models}

The introductory remarks stressed the importance of choosing the right structure of mathematical models, and this can hardly be overemphasized. It is absolutely essential to identify the right structure of a model, so that it is not left to the parameter estimation in a model to try to cover up for the wrong structure.

Another classical example which is often mistreated is the single component vapour pressure, which at very low pressures is modeled very close to the ideal Clausius-Clapeyron equation:

$$
p=\exp (a+b / T)
$$

The applicability of this model deteriorates as the pressure and temperature increases ( $b$ is always negative) and the deviation from the Clausius-Clapeyron equation is modified by the Antoine's model, which introduces another parameter $c$ and uses the temperature $t$ in degrees centigrade:

$$
p=\exp (a+b /(c+t))
$$

This mathematical form shows that the structure of the logarithm to the vapour pressure is basically a constant, then a proportionality to $t$ and then finally a slight 
hyperbolic curvature. The parameters may be transformed accordingly:

$$
\begin{gathered}
p=\exp \left(a^{\prime}+b^{\prime} t /\left(1+c^{\prime} t\right)\right) \\
a^{\prime}=a+b / c, \quad b^{\prime}=-b / c^{2}, \quad c^{\prime}=1 / c
\end{gathered}
$$

This transformation removes as much as possible of the undesirable correlation between the parameters in the model, and lets each term in the exponent be explained by a constant, a linear slope and a hyperbolic curvature. The modeling of the exponent as a polynomial is certainly not correct, but it is also clearly seen that it will be very hard to distinguish between a hyperbola and a second order term in the polynomial for a limited range of observation in $t$. The two forms:

$$
\exp \left(a^{\prime}+b^{\prime} t /\left(1+c^{\prime} t\right)\right) \quad \text { and } \exp \left(a^{\prime}+b^{\prime} t-c^{\prime \prime} t^{2}\right), \quad c^{\prime \prime}=c^{\prime} b^{\prime}
$$

are hardly distinguishable over a limited range in temperature, but they become dramatically different outside the region of $t$ used for the determination of $a^{\prime}, b^{\prime}$ and $c^{\prime}$. Hence, it is important to select the right mathematical structure, which is usually derived from the basic physical principles.

It is not only the single variable mathematical structure which is important, it is certainly equally important to consider the network structure of the cause and effect relationships. In process engineering, there are five very essential structures which will cover the majority of process networks and flow-sheets. Those are:

(1) The sequential or serial structure.

(2) The feed forward or the parallel structure.

(3) The feed back or recycle structure.

(4) The co-current structure.

(5) The counter-current structure.

The block diagram representation of these structures are shown in figures 1, 2 and 3 . These block diagrams have an analogous mathematical formulation, which has orders of magnitude difference in difficulty of solution and simulation. Typically, the mathematical formulation for the five different structures are of an explicit, semiimplicit and two-point boundary value nature.

Serial (sequential) structure

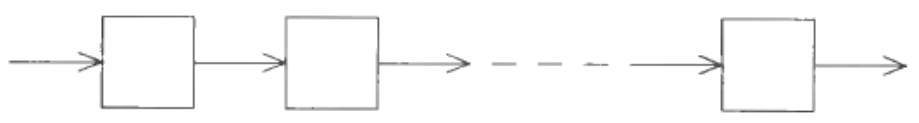

Combination of Serial and Parallel (feed-forward) structure

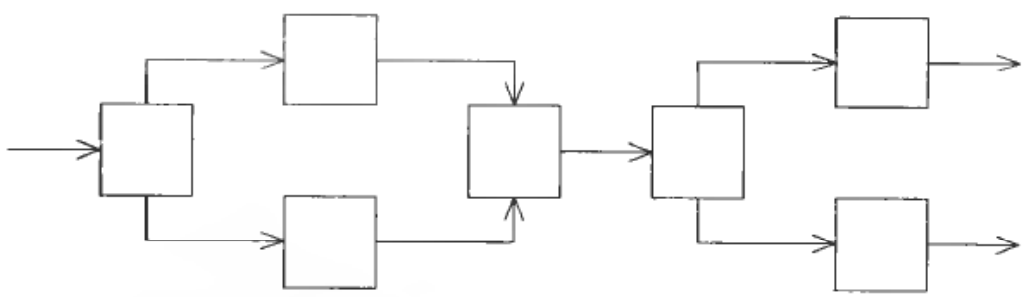

Figure 1. The serial and parallel (feed-forward) structure. 
This structure is crucial to the following situations:

1. Feed-back Control

2. Recycles and Recovery

3. Physical and Process Variable interactions

4. Iterative Solution of Equations

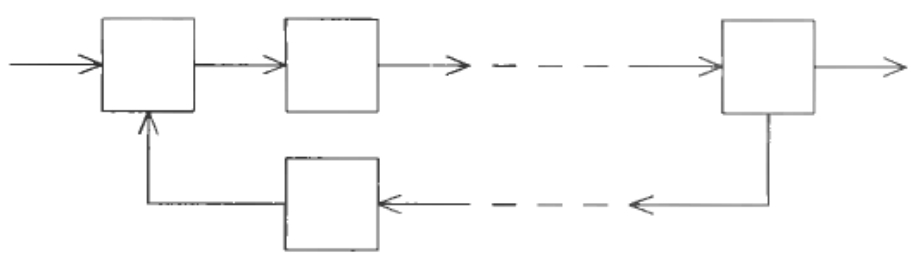

Figure 2. The feed-back structure.

The serial structure is explicit, solved by direct substitution or Picard iteration:

$$
\mathbf{x}_{i}=\mathbf{f}_{i}\left(\mathbf{x}_{i-1}\right)
$$

The parallel structure is also explicit, but solved by direct substitution in two parallel operations:

$$
\begin{aligned}
\mathbf{x}_{i} & =\mathbf{f}_{i}\left(\mathbf{x}_{i-1}\right) \\
\mathbf{x}_{i}^{\prime} & =\mathbf{f}_{i}^{\prime}\left(\mathbf{x}_{i-1}\right) \\
\mathbf{x}_{i+1} & =\mathbf{f}_{i+1}\left(\mathbf{x}_{i}, \mathbf{x}_{i}^{\prime}\right)
\end{aligned}
$$

The feedback structure is always of a semi-implicit nature, as the output is a function of itself:

$$
\begin{aligned}
& \mathbf{x}_{i}^{\prime}=\mathbf{f}_{i}^{\prime}\left(\mathbf{x}_{i-1}, \mathbf{x}_{i}\right) \\
& \mathbf{x}_{i}=\mathbf{f}_{i}\left(\mathbf{x}_{i}^{\prime}\right)
\end{aligned}
$$

A Co-Current problem is an initial boundary value problem.

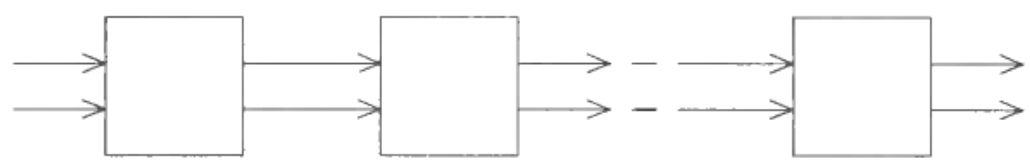

A Counter-Current problem is a two point boundary value problem which has a very large number of feed-back loops.

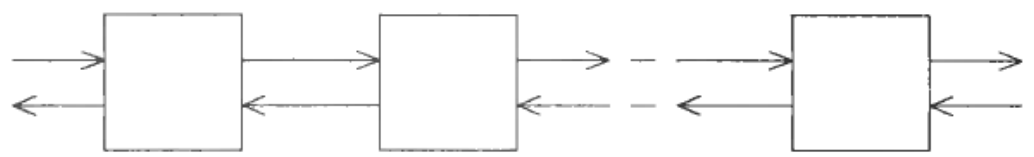

Figure 3. The co-current and counter-current structure. 
This structure must always be solved by interation, and the analogy between feedback control and a feedback or semi-implicit mathematical structure is obvious, as it has been recognized in numerical analysis for a long time.

It should be noted, however, that the feedback structure may be calculated without any iterations, if some conditions are met. Suppose the input may be calculated from the output, which is the same as reversing the flow of information in the network. Then the whole calculation may be reversed:

$$
\begin{aligned}
\mathbf{x}_{i}^{\prime} & =\mathbf{g}_{i}\left(\mathbf{x}_{i}\right) \\
\mathbf{x}_{i-1} & =\mathbf{g}_{i}^{\prime}\left(\mathbf{x}_{i}, \mathbf{x}_{i}^{\prime}\right)
\end{aligned}
$$

which has converted the feedback structure to a non-iterative parallel structure of explicit form. There are several examples from reactor simulation of cascaded tanks where this principle is applied with great ease and success (Asbjørnsen, 1974).

The co-current principle is really a serial structure with two parallel stream variables, but each stage is intermingling the two phase variables in a semi-implicit way:

$$
\begin{aligned}
& \mathbf{x}_{1, i}=\mathbf{f}_{1, i}\left(\mathbf{x}_{1, i}, \mathbf{x}_{2, i}, \mathbf{x}_{1, i-1}\right) \\
& \mathbf{x}_{2, i}=\mathbf{f}_{2, i}\left(\mathbf{x}_{1, i}, \mathbf{x}_{2, i}, \mathbf{x}_{2, i-1}\right)
\end{aligned}
$$

Hence, each stage may be simulated or calculated by local iteration if one proceeds from the start where $\mathbf{x}_{10}$ and $\mathbf{x}_{20}$ are given. This structure may also benefit from a reverse calculation, if the input may be calculated from the outputs.

The counter-current structure is by far the most complicated in process engineering, as it involves the solution of a two point boundary problem. The mathematical relations are now:

$$
\begin{aligned}
& \mathbf{x}_{1, i}=\mathbf{f}_{1, i}\left(\mathbf{x}_{1, i}, \mathbf{x}_{2, i}, \mathbf{x}_{1, i-1}\right) \\
& \mathbf{x}_{2, i}=\mathbf{f}_{2 . i}\left(\mathbf{x}_{1, i}, \mathbf{x}_{2, i}, \mathbf{x}_{2, i+1}\right)
\end{aligned}
$$

and a reverse information processing does not help because $\mathbf{x}_{10}$ is given at $i=1$ and $\mathbf{x}_{2, n+1}$ is given at $i=n$. This problem is therefore solved as a general two-point boundary value problem, where one iterates on the boundary conditions or on the size of the apparatus (invariant imbedding) or both.

In practically all exchange operations, it turns out that the counter-current mode is the optimal and hence the most attractive. The counter-current structure is therefore very essential and should be formulated and studied as such. A closer comparison, shows that the counter-current structure is really a feed-back structure also with very many recycle loops connected together. That is the reason why all tubular reactors modeled as stirred tanks with back-mixing will be recognized as a countercurrent structure. This back-mixing acts as a counter-current flow to the main flow, but also as a recycle stream. That is also the reason why distillation columns may be treated as a counter-current operation (Holland, 1963), but also as a recycle process with very many recycle loops (Kapoor, McAvoy and Marlin, 1985).

\section{The conservation principle for scalar variables}

The conservation principle is a general basis for all process modeling, and may be extended to any system where a balance calculation of rates is made. In its most general form, the conservation principle is applied as a balance of rates for a given system with defined boundaries. Define the rates: 
$r_{a}=$ Rate of total accumulation within the system

$r_{i}=$ Rate of total inflow to the system

$r_{e}=$ Rate of total effluent flow from the system

$r_{p}=$ Net rate of production within the system

Then the conservation principle is simply a balance of rates:

$$
r_{a}=r_{i}-r_{e}+r_{p}
$$

always expressed as extensive variables per unit time, like component materials, energy, mass, cash, etc.

Scalar quantities like component materials and energy may be expressed as extensive variable in terms of a product of a basic extensive variable like mass, volume or total moles, and an intensive variable. As the basis for the intensive variables, volume, mass or mole may be chosen. The mass basis is the most general one and usually invariant to intensive variables, like pressure, temperature and concentrations. Even if it does not comply with some of the older conventions in chemical engineering, a mass basis is chosen throughout. This is in line with modern fundamental textbooks (Aris, 1965).

For a given system volume, or enclosure, the conservation principle may be written for a general, scalar extensive variable, expressed as a product of a basic extensive variable and an intensive variable:

$$
\frac{d}{d t}\left[\int_{M} i d M\right]_{a}=(m i)_{i}-(m i)_{e}+\left[\int_{M} r d M\right]_{p}
$$

where the intensive net rate of production $r$ is taken on a mass basis.

If the rate of production is a first order process, regardless of chemical process or otherwise, where $r$ is proportional to $i$, i.e. $r=k(t) i(t)$, at any point within the system, the total production and accumulation rate may be introduced as a new variable, by the use of a Lagrange multiplier:

$$
\frac{1}{\lambda(t)} \frac{d}{d t}\left[\lambda(t) \int_{M} i d M\right]=(m i)_{i}-(m i)_{e}
$$

where the Lagrange multiplier is the function $\lambda(t)$ in general defined as the expontential function:

$$
\hat{\lambda}(t)=\exp \left(-\int_{0}^{t}\left(\int_{M} r d M / \int_{M} i d M\right) d \theta\right)=\exp \left(-\int_{0}^{t} k(\theta) d \theta\right)
$$

This allows a solution of the total accumulation of the property in question and within the total system mass:

$$
\int_{M} i(t) d M=\frac{1}{\lambda(t)}\left[\int_{M} i(0) d M+\int_{0}^{t} \lambda(\theta)\left((m i)_{i}-(m i)_{e}\right) d \theta\right]
$$

A useful and general conclusion from this observation, is that if the inlet and outlet extensive flows are measured, and the rate constant is given as a function of time, it is possible to calculate the amount of accumulation within the system at any time, and regardless of the system extension. This is exactly analogous to the observation in reaction engineering, that the residence time distribution is sufficient information for the calculation of total conversion for first order processes (Denbigh 1964). 
If the rate process is of first order and with known rate constant, the total accumulation of a property within the system is determined uniquely by the input and output extensive flows.

The input feed rate to the system is usually known, but the total outlet effluent rate is dependent on the intensive variable within the system. If eqn. (31) is used for a design purpose where measurements of the outlet are not available, it is necessary to know the flow pattern within the system to be able to solve the overall, dynamic conservation principle. Some of the less common examples of application are given below.

\subsection{The conservation principle applied to cash flow}

If the extensive quantity is considered to be cash within a company (the total system), the distribution of cash within the company is immaterial. Assume that the cash is always invested such that it carries interest, which may be time varying, but which is the same for all cash. Then the conservation principle for cash is simply:

$$
d C / d t=(m i)_{i}-(m i)_{e}+r C=c(t)+r C
$$

where the extensive cash inflow is a product of sales volume $m$ (extensive flow) and unit price $i$ (intensive) and the cash outflow is a product of purchase volume of products, utilities or services (extensive flow) and their unit prices (intensive). The difference between those constitutes the net cash flow $c(t)$.

The interest rate $r$ is the real interest rate, which is the difference between capital gains by bank interest compounding and capital loss by inflation. The conservation principle for cash is seen to be easily solved in this case, as an integration gives the company cash at any time:

$$
C(t)=\left[C(0)+\int_{0}^{t} \lambda(\theta) c(\theta) d \theta\right] / \lambda(t)
$$

where the Lagrange multiplier is equivalent to a discount factor which again is seen to be related to the average real interest rate:

$$
\lambda(t)=\exp \left(-\int_{0}^{t} r(\theta) d \theta\right)
$$

as in the general case of conservation. The application of a differential cash conservation implies continuous compounding.

When this result is inserted into the solution, eqn. (33) expresses the general discounted cash flow formula with continuous compounding of the real interest giving the company cash at any time:

$$
C(t)=C(0) \exp \left(\int_{0}^{t} r(\theta) d \theta\right)+\int_{0}^{t} c(\theta) \exp \left(\int_{\theta}^{t} r(\vartheta) d \vartheta\right) d \theta
$$

The analogy between the economics example and the physical example in (eqn. (31) with distributed rate of production, would be a portfolio of investments where the net interest rate will be different for the different investments. In both cases, the distribution of accumulation and production rates within the system is necessary. However, the example shows a direct and well known applicability of the physical conservation principle to the dynamics of economics. As such, the example serves the purpose at demonstrating the usefulness of general principles and the applicabil- 
ity of a systems' engineering approach to modeling. Dynamic modeling tools for the conservation in physical systems may therefore be applied to economics with ease.

\subsection{The ideal mixing stage for single phases}

The flow pattern within a completely mixed tank is that of a total isotropy and homogeneity. In this case, the intensive properties are the same within the total system, independent of position. The conservation and production within the system may then be integrated easily. This gives:

$$
\frac{d}{d t}(\rho V i)_{e}=(m i)_{i}-(m i)_{e}+(r V \rho)_{e}
$$

where $\rho$ is the homogeneous density and $V$ the system volume, which may very well vary with time.

An often overlooked and general identity which simplifies the equation above, is the total mass balance:

$$
\frac{d}{d t}(\rho V)=m_{i}-m_{e}
$$

which gives a general equation for the dynamics of the intensive properties of a mixing stage:

$$
\frac{d i}{d t}=s_{i}\left(i_{i}-i\right)+r
$$

where $s_{i}$ is the feed space velocity on a mass basis.

For all first order production processes, where $r$ is proportional to $i$, eqn. (39) is readily solvable by integration:

$$
i(t)=\left[i(0)+\int_{0}^{t} \lambda(\theta) s_{i}(\theta) i_{i}(\theta) d \theta\right] / \lambda(t)
$$

where the Lagrange multiplier is related to the production rate constant as well as the space velocity:

$$
\lambda(t)=\exp \left(\int\left(k(\theta)-s_{i}(\theta)\right) d \theta\right)
$$

in a way very similar to the cash flow analysis and dynamics. The rate constant $k(t)$ is analogous to the compound interest rate in eqn. (32).

\subsection{A single tank with multi-component, multiple feed streams}

Simple mixing stages, where intensive variables are to be maintained at a desired level, occur frequently. For simplicity, the production rate is assumed zero, and the dynamics of the intensive variables are described by:

$$
\frac{d}{d t} i^{k}=\sum s_{i}^{j}\left(i_{i, j}^{k}-i^{k}\right)
$$

where $i_{i, j}^{k}$ is the intensive variable number $k$ of the input feed stream number $j$. All these inlet variables form a matrix $S$ of dimension $n \times m$ where $n$ is the number of 
intensive variables and $m$ is the number of input streams. Hence, one may write eqn. (42) in integrated form with a compact vector notation:

$$
\mathbf{i}(t)=\left[\mathbf{i}(0)+\int_{0}^{t} \lambda(\theta) S(\theta) \mathbf{s}_{i}(\theta) d \theta\right] / \lambda(t)
$$

where $\mathbf{s}_{i}(\theta)$ is the vector of space velocities, and the Lagrange multiplier $\lambda(\theta)$ is as traditional:

$$
\lambda(t)=\exp \left(-\int_{0}^{t} \sum_{j=1}^{m} s_{i}^{j}(\theta) d \theta\right)=\exp \left(-\int_{0}^{t} s(\theta) d \theta\right)
$$

and $s(\theta)$ is the sum of all space velocities.

If it is desirable to keep the intensive variables at a given level, say a set-point, the conservation principle gives the ideal balance control of the space velocities of the various inlet streams. The simple differential equation expressing the conservation principle may be written in vector form:

$$
d \mathbf{i} / d t=\left(S-\mathbf{i e}^{T}\right) \mathbf{S}
$$

This equation suggests a simple balance control of the space velocities which would make the rate of change of the intensive variable theoretically zero. This leads to a general algorithm which is referred to as extensive variable balance control:

$$
\left(S-\mathbf{i e}^{T}\right) \mathbf{s}=\mathbf{0}
$$

One of the space velocities will have to be specified, and this is normally done by specifying the sum of the inlet space velocities equal to the outlet space velocity. This attempts to satisfy the total mass balance:

$$
\mathbf{e}^{T} \mathbf{s}=m_{e} /(\rho V)=s_{e}
$$

Hence, in order for eqn. (45) to be non-singular together with eqn. (45), one would require that $m \geqslant n+1$ and the total matrix $S^{\prime}$ to be non-singular.

$$
S^{\prime}=\left[\begin{array}{c}
S-\mathbf{i e}^{T} \\
\hdashline \mathbf{e}^{T^{-}}
\end{array}\right]
$$

The space velocities are then computed according to the simple formula:

$$
\mathbf{s}=\left(S^{\prime}\right)^{-1}\left[\begin{array}{c}
\mathbf{0} \\
-S_{e}
\end{array}\right]
$$

This result is somewhat theoretical, because the practical application may be questionable. It requires knowledge or estimation of the matrix $S$ and the vector $i$. In some cases this may be obtained by measurements and some assumptions of absence of a component in a feedstream (corresponding element in $S$ equal to zero). In practice, however, estimation or measurement of the coefficients in the matrix, i.e. the intensive properties of the feeds, may not be justified from an economic point of view.

If the material balance is insufficient or the coefficients given with errors, the simple feed forward control law in eqn (48) will not give $d \mathbf{i} / d t=0$, but a slow drift in the states $i$. Therefore, the extensive variable balance control is usually modified by an additive term from a feed back of the intensive variable $i$ in the tank effluent. If a 
simple proportional feed back control is suggested, in addition to the feed forward control, the total model for the closed loop control will be:

$$
\frac{d \mathbf{i}}{d t}=\left(S-\mathbf{i e}^{T}\right)\left[\left(S^{1}\right)^{-1}\left[\begin{array}{l}
0 \\
s_{e}
\end{array}\right]+K\left(\mathbf{i}_{s p}-\mathbf{i}\right)\right]
$$

which turns out to be a non-linear (quadratic or bi-linear) differential equation in the inputs and outputs. This feed forward control is perfect for disturbance rejection, but requires an investment in instrumentation of the inputs. The quality of this control, its stability, regulator performance and disturbance rejection ability may be investigated by a linearization or a full simulation, but it would be much more appropriate to formulate an economic objective function for the control including the process constraints and perform optimal control.

\section{Conservation principle and stoichiometry}

The Avogadro's discovery of the stoichiometric numbers of chemical reactions was only another example on the general conservation principle, this time applied to atoms and molecular structures. In an extention of linear algebra to incorporate letters and integers, the atomic formula for a molecule may be written:

$$
\mathbf{m}=A \mathbf{a}
$$

where $A$ is a matrix of the number of atoms required to build a molecule, the vector $\mathbf{m}$ a vector of chemical species formulae and the vector a the letter symbols of the atomic elements. Consider alcohol as an example:

$$
\begin{gathered}
m=\mathrm{C}_{2} \mathrm{H}_{5} \mathrm{OH}=2 \mathrm{C}+6 \mathrm{H}+1 \mathrm{O} \\
\mathbf{a}^{\mathrm{T}}=[\mathrm{C}, \quad \mathrm{H}, \quad \mathrm{O}] ; \quad A=\left[\begin{array}{lll}
2, & 6, & 1
\end{array}\right]
\end{gathered}
$$

The stoichiometry turns out to be a conservation principle for atoms, as the conservation of atoms is totally independent of the extent of reactions. In matrix form, this is written:

$$
N^{\mathrm{T}} \mathbf{m}=\mathrm{O} \quad \text { and } \quad N^{T} A \mathbf{a}=\mathrm{O}
$$

which means that the atomic matrix $A$ and the stoichiometric matrix $N^{T}$ are always orthogonal. Take as an example, steam reforming of methane:

$$
\begin{aligned}
& \mathrm{CH}_{4}-\mathrm{H}_{2} \mathrm{O}+3 \mathrm{H}_{2}+\mathrm{CO}=0 \\
-\mathrm{CO}-\mathrm{H}_{2} \mathrm{O}+\mathrm{H}_{2}+\mathrm{CO}_{2}=0 & \\
N^{T}= & {\left[\begin{array}{rrrrr}
-1 & -1 & 3 & 1 & 0 \\
0 & -1 & 1 & -1 & 1
\end{array}\right] } \\
\mathbf{m}^{T}= & {\left[\begin{array}{lllll}
\mathrm{CH}_{4}, & \mathrm{H}_{2} \mathrm{O}, & \mathrm{H}_{2}, & \mathrm{CO}, & \mathrm{CO}_{2}
\end{array}\right] } \\
A^{T}= & {\left[\begin{array}{lllll}
1 & 0 & 0 & 1 & 1 \\
4 & 2 & 2 & 0 & 0 \\
0 & 1 & 0 & 1 & 2
\end{array}\right] ; \quad N^{T} A=0 ; \quad A^{T} N=0 } \\
\mathbf{a}^{T}= & {\left[\begin{array}{lll}
\mathrm{C}, & \mathrm{H}, & \mathrm{O}
\end{array}\right] }
\end{aligned}
$$


The general conclusion that may be drawn from this observation, is that the rank of the stoichiometric matrix is sufficient to determine the number of reactions with independent stoichiometries, an observation often neglected in process modeling. Let this number be $m$ and let the number of components be $n$. Then a number of $n-m$ reaction invariant relations may be written, expressing $n-m$ reaction invariant conservations. The conservations of atoms are examples on such invariant relations.

As it may become apparent, the concepts of stoichiometry and reaction invariance and the concepts of dimensional analysis and unit invariance are practically identical, they are just applications of general vector decomposition in linear algebra.

\section{Stoichiometry and the reaction rate}

The chemical reactions are the heart of process engineering as they affect the composition of the product streams which are the basis for the production net cash flow. The chemical reactions define a reaction scheme or stoichiometry of components and the reaction rates define the rate at which the reactions proceed. If such rates are defined per unit of mass of the fluid moving through the reactor, let those reaction rates be grouped into a vector $\boldsymbol{r}$. Then the chemical component production rates are:

$$
\mathbf{r}_{p}=N \mathbf{r}
$$

Premultiplying with a matrix $A^{T}, A$ being a full rank matrix of dimension $n \times(n-m)$ where $m$ is the rank of the matrix $N, A$ is chosen such that $N^{T} A=0$. This means that a subset of linear combinations of the production rates $\mathbf{r}_{p}$ are zero, and behave as if no reaction took place, they are reaction invariant.

$$
A^{T} \mathbf{r}_{p}=A^{T} N \mathbf{r}=\mathbf{0}
$$

If the matrices $A$ and $N$ are partitioned as:

$$
N^{T}=\left[\begin{array}{c:c}
N_{11} & N_{12} \\
\hdashline N_{21} & N_{22}
\end{array}\right] ; \quad A=\left[\begin{array}{c}
A_{1} \\
\hdashline A_{2}
\end{array}\right]
$$

where $N_{11}$ is the square, non-singular part of $N$ of dimension $m \times m$ and $A_{2}$ a square, non-singular part of $A$ of dimension $(n-m) \times(n-m)$. Then, the matrix $A_{1}$ is simply:

$$
A_{1}=-N_{11}^{-1} N_{12} A_{2} \quad \text { or } A_{1}=-N_{11}^{-1} N_{12} \quad \text { if } \quad A_{2}=I
$$

since:

$$
N_{22}-N_{21} N_{11}^{-1} N_{12}=0
$$

if $N$ has reduced rank. A good example of stoichiometry with reduced rank is in the pyrolysis of an ethane mixture (Villadsen et al., 1977). The stoichiometric matrix may then be written as a product of two full rank matrices (of rank $m$ ):

$$
N^{T}=B C=\left[\begin{array}{c}
B_{1} \\
\hdashline B_{2}
\end{array}\right]\left[C_{1}: C_{2}\right]
$$

where:

$$
B_{1}=N_{11}, \quad B_{2}=N_{21}, \quad C_{1}=I \quad \text { and } \quad C_{2}=N_{11}^{-1} N_{12}
$$


Then, some of the reaction rates in $\boldsymbol{r}$ may be written as linear combinations of a set of basic reaction rates $\boldsymbol{r}_{b}, \boldsymbol{r}=L \boldsymbol{r}_{b}$. The matrix $L$ is then the left pseudo-inverse of $C$ :

$$
L=\left[\begin{array}{c}
N_{11}^{T} \\
N_{12}^{\mathrm{T}}
\end{array}\right]\left[N_{11}^{T}+N_{11}^{-1}\left(N_{12} N_{12}^{T}\right)\right]^{-1}
$$

and the reaction rates and the production rates may be written in terms of this basic reaction rate:

$$
\begin{aligned}
\mathbf{r} & =L \mathbf{r}_{b} \\
\mathbf{r}_{p} & =N \mathbf{r}=N L \mathbf{r}_{b}=\left[\begin{array}{c}
N_{11} \\
\hdashline N_{21}^{-}
\end{array}\right] \mathbf{r}_{b}
\end{aligned}
$$

If the matrix $N$ has full rank, then the matrices are $B=N, C=I$ and $L=I$ and the reaction rates $\boldsymbol{r}$ are the true basic reaction rates $\boldsymbol{r}_{b}$, which is obvious and well known (Levenspiel, 1964).

\section{Reaction variants and invariants in chemical reactor modeling}

The chemical reactor is modeled as a combination of flow pattern models and reaction kinetic models. The flow pattern models comprise convective, conductive and radiation transport phenomena and describe the behaviour of the overall transport of extensive properties throughout the reactor. Since the chemical reactions are totally dependent on the presence of molecules at the sites of reaction, it is obvious that the transport phenomena and the flow patterns through the reactor are of the greatest importance. Flow patterns through a reactor are described on a molecular basis as residence time distributions or on a hydrodynamic basis as velocity distributions. Chemical reactor modeling logically starts with homogeneous reactors, which are the simplest, and where the reactions take place throughout every fluid element of the reactor.

\subsection{The homogeneous reactor}

The conservation principle may be written as a general operator on an intensive property, first without chemical reactions and then with chemical reactions. Such a general operator may be written (Asbjørnsen, 1972):

$$
L(i)=f
$$

where $f$ is zero or a forcing function.

For a stirred tank, the operator is shown to be:

$$
L=\left[\frac{d}{d t}+s(t)\right] \text { and } f=s(t) i_{f}(t)
$$

For a tubular reactor without any exchange of heat or materials with the environment, the operator is expressed as a partial differential operator in terms of fractions of the total volume $x$ :

and:

$$
L=\left[\frac{\partial}{\partial t}+s(x, t) \frac{\partial}{\partial x}\left(1-E(x, t) \frac{\partial}{\partial x}\right)\right]
$$

$$
f=0
$$


where the apparent dimensionless axial dispension $E$ may be different for the various intensive variables. The forcing functions in this particular case are entering as boundary conditions at both ends of the tube:

$$
s(x, t)\left[1-E(x, t) \frac{\partial}{\partial x}\right] i=s_{f}(t) i_{f}(t) ; \quad x=0
$$

at the entrance of the reactor and:

$$
E(x, t) \frac{\partial}{\partial x} i=0 ; \quad x=1
$$

at the exit of the reactor.

The solution of the conservation operator without chemical reactions shows how the various intensive properties propagate through the reactor without any chemical reactions. The propagation of the same properties with chemical reactions, requires another term for its solution, the net production term. As shown for a special case of the stirred tank, the conservation principle for a stirred tank reactor may be written:

$$
\frac{d}{d t} \mathbf{i}=s(t)\left(\mathbf{i}_{f}-\mathbf{i}\right)+N \mathbf{r}
$$

or in terms of a general differential operator:

$$
L(\mathbf{i})=\mathbf{f}+N \mathbf{r}
$$

Multiplying with the orthogonal matrix $A^{T}$, shows that $A^{T} \mathbf{i}$ propagate through the reactor as a true reaction invariant, since $A^{T} N=0$ and the differential operator $L$ is assumed to be linear:

$$
L\left(A^{T} \mathbf{i}\right)=A^{T} \mathbf{f}
$$

However, the variable $\eta=\left(B^{T} B\right)^{-1} B^{T} \mathbf{i}=B^{+} \mathbf{i}$ propagates through the reactor as a true reaction variant, as now:

$$
L(\boldsymbol{\eta})=B^{+} \mathbf{f}+\mathbf{r}_{b}
$$

The variable $\boldsymbol{\eta}$ is usually referred to as an extent of the reactions, as it is seen to be controlled entirely by the basic reaction rate $\mathbf{r}_{b}$.

The logical extension of these modeling ideas, to a general linear operator for homogeneous reactors, shows how valuable a more general systematic approach to modeling is. Since the conservation operator for the fluid flow through the reactor is a differential operator operating on a vector of intensive properties, an orthogonal decomposition of the variables is useful both for modeling and for parameter estimation. For example, the tubular reactor with axial dispersion will have a general structure of its decomposed model:

$$
L\left(A^{T} \mathbf{i}\right)=0
$$

and:

$$
L\left(B^{+} \mathbf{i}\right)=\mathbf{r}_{b}
$$

Consequently, the model for the reaction invariants $A^{T} \mathbf{i}$ may be used for an independent estimation of the flow pattern through the reactor at the time of the reac- 
tions, while the extents of the reactions $B^{+} \mathbf{i}$ may be used for the estimation of the reaction kinetic parameters. In view of the complications usually encountered in parameter estimation of chemical reactors, orthogonal decompositions of the variables as indicated here, should indeed be applied to a greater extent than is normal practice today.

Orthogonal decomposition and general simplifications of reactor modeling have been known and applied in certain simulation studies (Asbjørnsen, 1982), but it is surprising to see how little emphasis has been given to these facts in reaction engineering books and papers. As it is shown here, it all relates to fundamental properties of linear algebra, concerning base decomposition, and ranks of matrices.

\subsection{The heterogeneous reactor}

Fixed bed catalytic reactors differ significantly from homogeneous reactors, and still, they are the simplest possible extensions of a homogeneous reactor model. the fluid is treated as a single homogeneous phase, with an interface to the catalyst. At this interface, there are transports of extensive properties from the fluid to the solid surface of the catalyst. To a certain extent, the catalyst phase has an ability to accumulate the extensive properties, particularly heat. The modeling of the reactor usually distinguishes between the two phases, where the link between the phases are the transport phenomena on the fluid-solid interface.

The homogeneous phase is modeled as a homogeneous reactor, but the interface transport takes the place of the homogeneous reaction rate. As an example, consider the tubular reactor (the packed bed reactor):

$$
L(\mathbf{i})=a \mathbf{r}_{t}
$$

where the rate vector $\mathbf{r}_{t}$ is the transport flux of extensive properties across the interface and $a$ is a parameter expressing interfacial area per unit of volume of the reactor. The transport flux is usually modeled by a simple boundary layer model with an intensive property difference as a driving force and an empirical transport or transfer coefficient, which are different for the different properties:

$$
\mathbf{r}_{\mathbf{t}}=K \Delta \mathbf{i}
$$

The driving force $\Delta \mathbf{i}$ is a difference of an intensive variable in the bulk of the fluid and the catalyst interface, and $K$ is a matrix (usually diagonal) of transport numbers for the interface boundary layer.

If the catalyst particles have the ability to accumulate the extensive properties, this must be taken into account. The simplest possible model for such a phenomenon, would be to consider the particle as a perfect conductor of the property in question (no gradients inside the catalyst particle). The actual transport flux will now be the input variable to a simple 'stirred tank' modeling approach to the catalyst particle:

$$
\frac{d}{d t}\left(\mathbf{i}_{p}\right)=S\left(\mathbf{i}-\mathbf{i}_{p}\right)+N \mathbf{r}
$$

where the matrix $S$ is a matrix of transport properties per unit weight of the catalyst. This matrix is usually considered to be a diagonal.

Multiplying this equation again with $A^{T}$, removes the dependence on the cata- 
lytic reaction rate $\mathbf{r}$, as one may now write the model relations for the reaction invariant part of the state variables in the catalyst particle:

$$
\frac{d}{d t}\left(A^{T} \mathbf{i}_{p}\right)=A^{T} S\left(\mathbf{i}-\mathbf{i}_{p}\right)
$$

but it is quite clear that the variable $A^{T} \mathbf{i}$ in the homogeneous reactor or $A^{T} \mathbf{i}_{p}$ at the catalyst surface are no longer reaction invariants.

However, there is a special case where the concept of reaction invariance applies equally well to heterogeneous reactors, and that is when $S$ has reduced rank equal to the rank of, $(n-m)$. In that case, one may write $S=B A^{T}$ and compute the matrix $B$ :

$$
B=\left[\begin{array}{l}
S_{11} \\
\hdashline S_{21}^{-}
\end{array}\right] A_{1}^{-1}
$$

but the two remaining matrices in $S$ must obey the relationship:

$$
S_{12}=S_{11} A_{1}^{-1} A_{2} \text { and } S_{22}=S_{21} A_{1}^{-1} A_{2}
$$

where it is seen that the last condition is automatically satisfied if the rank of the matrix $S$ is $m$, since then:

$$
S_{22}=S_{21} S_{11}^{-1} S_{12}
$$

In this particular case, a reduced set of the transport phenomena are indeed reaction invariant, since:

$$
\frac{d}{d t}\left(A^{T_{\mathbf{p}}}\right)=\left(A^{T} B\right)\left(A^{T} \mathbf{i}-A^{T_{i}}\right)
$$

but this situation is very unlikely in practice.

The general conclusion is therefore, that heterogeneity and transport phenomena ruin the concept of reaction invariance or the general concepts of assymptotic invariance. (Fjeld, Asbjørnsen and Åstrøm, 1974). For a simulation of a heterogeneous reactor, it is not possible to decompose the state variables in the reaction variant and invariant sets, the transport phenomena introduce other types of couplings and relations between the variables, recognized as the chromatographic effects (Amundsen and Aris, 1973). These effects make the residence time distributions different for the various components as utilized in chromatographic separation. As it may have been noticed from the operator eqn.(71) on the reactor invariants, that was one of the conditions why the concept of reaction invariance applied to homogeneous systems. The residence time distribution was the same for all reaction invariant variables. An even more general conclusion is therefore that the concepts of reaction invariance are inapplicable when the residence time distributions are different for the various components participating in the reactions.

\section{REFERENCES}

Amundoson, N. R. and ARIS, R. (1973). Mathematical Methods in Chemical Engineering, Vol. 2, First Order Partial Differential Equations with Applications. (Prentice-Hall, Inc., Englewood Cliffs, New Jersey).

ARIS, R. (1965). Introduction to the Analysis of Chemical Reactors (Prentice-Hall, New York). Asbugrnsen, O. A. (1966). Process Control Laboratory Experiments (University of The West Indies, Trinidad \& Tobago). 
AsBJøRnSEn, O. A. 1972). Reaction invariants in chemical reactor dynamics. Kemian Teollisus, Nr. 10, 633-639.

Asbjornsen, O. A. and Hertzberg, T. (1974). Constrained regression in chemical engineering practice, Chemical Engineering Science, 29, 679-687.

Asbuørnsen, O. A. (1974). Process Dynamics (Åbo Akademi, Turku).

Asbugrnsen, O. A. (1975). Process Control, (Norsk Hydro a.s., Porsgrunn). (In Norwegian).

ASBJORNSEN, O. A. (1982). Modelling and simulation in computer aided design and operation of chemical plants, Kemia-Kemi, 9, 103-107.

Denbigh, K. (1964). Principles of Chemical Equilibrium (Cambridge University Press, Cambridge).

FJeld, M. AsbJørnsen, O. A., and ÅstrøM, K. J. (1974). Reaction invariants and their importance in the analysis of eigenvectors, state observability and controllability of the continuous stirred tank reactor. Chemical Engineering Science, 29, 1917-1926.

HimmelblaU, D. M. (1978). Fault Detection and Diagnosis in Chemical and Petrochemical Processes. (Elsevier Sci. Publ. Co. Amsterdam-Oxford-New York).

Holland, C. D. (1963). Multicomponent Distillation (Prentice-Hall Inc., Englewood Cliffs, New Jersey).

KAPOOR, N., MCAvoY, T. J., and MaRLIN T. E. (1986). Effect of recycle structure on distillation tower time constants, AIChE Journal, (In press).

LeVensPiel, O. (1964). Chemical Reaction Engineering (John Wiley, New York).

NorSK Hydro A.S. (1980), Personal Communication, Porsgrunn, Norway.

OhNo, H., and NaKANISHI E. (1985). Dynamic Resiliency of Heat-Integrated Distillation Systems. E.F.ChE Publ. Series, No 40 (The Institution of Chemical Engineers, London) pp. 469-480.

ShInSKey, F. G. (1967). Process Control Systems (McGraw-Hill, New York).

Villadsen, J. V., Asbiørnsen, O. A., Harg, K. E., Heyerdahl, N., and Harg, M. (1977). Simulation of an industrial ethylene cracker, a comparison between the stirred tank and the differential equation approach. In Computer Application in the Analysis of Chemical Data and Plants, Proceedings from Chem Data '77, Helsinki. (Science Press, Princeton) pp. 27-41. 